\title{
Reduction in resource utilization and increase in patient satisfaction two years after implementation of Perioperative Surgical Home
}

\author{
Devanand Mangar ${ }^{1,2,3}$, Prachiti H. Dalvi ${ }^{1}$, Thomas Bernasek ${ }^{4}$, Enrico Camporesi*1,2,3 \\ ${ }^{1}$ TEAMHealth Anesthesia, Tampa, FL, United States \\ ${ }^{2}$ Tampa General Hospital, Tampa, FL, United States \\ ${ }^{3}$ University of South Florida, Tampa, FL, United States \\ ${ }^{4}$ Florida Orthopaedic Institute, Tampa, FL, United States
}

Received: November 17, 2016

DOI: $10.5430 /$ css.v3n2p18
Accepted: February 2, 2017

Online Published: March 22, 2017

\begin{abstract}
Introduction: Perioperative Surgical Home (PSH) provides coordinated, team-based care that is patient-centered, designed to guide patients efficiently through the surgical experience. We applied several changes to our anesthesia practice during 2014 and evaluated outcomes for all orthopedic major joint replacement surgeries completed in 2015. Since this was a quality improvement/utilization study, we were granted approval from our institutional review board to retrospectively review these data. Methods: We conducted a utilization review of all 1,356 patients who received total knee, hip, or shoulder joint replacement from 4 major surgical providers in 2015. Preoperative evaluation was limited and focused to each patient's unique medical conditions. Additionally, we reduced intraoperative fluid use, reduced continuous femoro-sciatic nerve or brachial plexus blocks by increasing the administration of single-shot regional blocks, limited transfusion, and minimized urinary catheter use. We improved pain consult response time and provided timely discharges.

Results: We noted 9\% reduction in preoperative imaging per case, $22 \%$ decrease in average number of tests per case, $87 \%$ fewer average units of red blood cell used per case, and a 0.4 day reduction in average length of stay (LOS). Patient satisfaction data showed constant improvement in pain management, and doctor communication.

Conclusions: Numerous small modifications to patient care collectively contributed to these progressive observed changes in patient outcomes from quarter to quarter.
\end{abstract}

Key Words: Perioperative Surgical Home, Patient outcomes, Perioperativists

\section{INTRODUCTION}

The Perioperative Surgical Home (PSH) is a patient-centered, team-based model of care coordination designed to help patients navigate their whole surgical experience, from the decision to have surgery to discharge from the hospital and return to function. ${ }^{[1]}$ The arrival of bundled payment pro- grams, such as the U.S. Centers for Medicare \& Medicaid Services (CMS) Comprehensive Care for Joint Replacement (CJR) model, requires physicians to adapt to new models of care delivery. ${ }^{[2]}$ The PSH model is a program developed by the American Society of Anesthesiologists (ASA) and Premier Inc. to focus on value while simultaneously increasing

\footnotetext{
*Correspondence: Enrico Camporesi; Email: ecampore@health.usf.edu; Address: TEAMHealth Anesthesia; Tampa General Hospital; University of South Florida, Tampa, FL, United States.
} 
patient satisfaction and reducing costs. ${ }^{[3,4]}$ The PSH model, analogous to the Medical Home model in the primary care practice setting, was proposed by the ASA as an innovative, patient-centered, surgical continuity of care model that incorporates shared decision making. ${ }^{[5]}$

After a rigorous review of the literature to plan and develop the process, the first phase of the PSH initiative was implemented from July 2014 through November 2015. Forty-four healthcare organizations from across the United States collaborated to establish and evaluate the PSH model during Phase One. ${ }^{[6]}$ Of these selected healthcare organizations, 73 percent launched a total of 64 pilot programs successfully and cared for thousands of patients. Our anesthesia service implemented this program to provide simplified and standardized pre-operative evaluation for all urology patients and demonstrated significant financial savings, unchanged patient outcomes and increased patient satisfaction. ${ }^{[7]}$ In 2015, we expanded this program to better suite new bundled payment plans such as the Bundled Payments for Care Improvement (BPCI) program, which applies to orthopedic surgery for major joint replacement.

\section{MATERIAls AND METHODS}

Though this was a quality improvement/assurance study, we were granted exemption from the institutional review board to review and analyze this quality data collected by our institution. Through a retrospective review of hospital quality data (Hospital Consumer Assessment of Healthcare Providers and Systems, HCAHPS) from Tampa General Hospital (TGH), we evaluated how resource utilization changed when receiving care through the PSH model. Further, we sought to understand how this model affected patient satisfaction.

\subsection{Patients}

We conducted a quality review of all patients who received total knee, hip, or shoulder joint replacement from 4 major surgical providers. At our institution, this entailed a quarterly review of billing information for 1,356 patients from January 1, 2015 to December 31, 2015.

\subsection{Preoperative clinical assessment}

Preoperative evaluation was personalized to each patient's unique medical conditions. Patients are seen by a physician assistant (PA) during their initial visit to the preoperative clinic. This dedicated PA works closely with attending anesthesiologists to comprehensively collect the patient's history. The following were used as indications for preoperative testing:

Published by Sciedu Press
(1) Complete blood count (CBC) for patients with history of anemia, hematuria, or cases where extensive blood loss is expected

(2) Basic metabolic panel (BMP) for patients with history of hypertension, renal disease, or electrolyte abnormalities

(3) Comprehensive metabolic panel (CMP) for patients with liver disease

(4) Prothrombin time (PT)/partial thromboplastin time (PTT)/international normalized ratio (INR) for patients on anticoagulant therapy or patients with liver disease

(5) Chest $x$-ray only on patients with current respiratory infection

(6) Electrocardiogram (EKG) on patients with recent symptoms of chest pain, shortness of breath, or on patients unable to complete $>4$ metabolic equivalents (METs) of activity

The preoperative clinical assessment intends to pinpoint patient-specific risks of surgery in an effort to better prepare the healthcare team to manage each patient. A critical component of this goal is optimizing the need for blood. This includes beta-blocker, statin, and anticoagulant therapy along with administration of subcutaneous recombinant erythropoietin and intravenous iron for preoperative anemia to minimize - or even eliminate - surgical allogeneic blood transfusions. Patient education and informed decision-making are also key aspects of the preoperative clinic: patients were provided detailed information about their surgical procedure and anesthesia to minimize anxiety and accelerate hospital discharge.

\subsection{Pain management}

An individualized pain management plan is outlined, encompassing preoperative oral medications and maintenance of the patient's chronic opioids. Preventative analgesia and decreased chronic post-surgical pain are stressed. Special attention is given to ensure timely pain consult responses by the Pain Management Team. We shifted to spinal anesthesia during the case followed by more supervised use of femoro-sciatic nerve blocks. Communication was critical in transitioning from spinal anesthesia to regional blocks or maintaining analgesia from regional blocks.

\subsection{Electronic medical record system}

To avoid redundant testing, imaging was transmitted and assembled preoperatively using a fully integrated electronic medical record system. No Foley catheters were used and patients were discharged as soon as applicable criteria were met. 


\subsection{Statistical analysis}

Data were averaged by quarter and a linear regression with time series component was applied to evaluate the trend over time. Forecast $\mathrm{R}$ package was used to assess significance.

\section{RESUlts}

With the implementation of the PSH model, our institution observed significant changes in Year 2 (2015). We performed a quarter by quarter evaluation of 1,356 patients ( 660 males and 696 females; average age $62.2 \mathrm{yr}$ ) who received a major orthopedic procedure. The percentage of patients classified as ASA 3 and ASA 4 (the most severe classifications) did not change significantly over the four quarters, making comparisons between the 4 quarters appropriate (see Table 1). The distribution of surgical procedures also remained consistent over the 4 quarters. In the first quarter (Q1), each case required 6.8 imaging tests. By Quarter $4(\mathrm{Q} 4)$, this number decreased to 6.1 images per case. Thus, from Q1 to Q4 average requirements for imaging tests decreased by $9 \%$. A more notable decrease of $22 \%$ was noted in number of tests per case: in Q1, 6.1 tests were ordered per case as compared to 4.7 tests per case ordered in $\mathrm{Q} 4$. Total transfused units of blood cells per quarter also visibly decreased from 39 in Q1 to 5 units in Q4, or an $87 \%$ decrease in number of blood units transfused. The change in average transfused units of blood approached significance when analyzed with Forecast $\mathrm{R}$ package $(p=.07)$ with a calculated slope coefficient of -16.7. Other measured variable regressions were not statistically significant. Of notice, however, we also report a reduction in length of stay (LOS) from 3.35 days in Q1 to 2.99 days in $\mathrm{Q} 4$. These data are summarized in Table 2 and visualized in Figure 1. Patient satisfaction data showed improvement in pain management and doctor communica- tion with a concurrent increase in survey response rate (see Figure 2).

\section{DiscuSSION}

The PSH model aims to improve surgical morbidity and mortality with limited resources. In this new model of healthcare delivery, anesthesiologists will assume the role of perioperative physicians and will play a critical role in promoting standardization of perioperative care. The comprehensive nature of the PSH model allows continuity of care and capitalizes on the team-based approach of coordinated perioperative care, to increase hospital efficiency and improve outcomes and patient satisfaction.

Despite the decreased use of resources and better patient outcomes reported in our study, our data have limitations that must be considered: LOS was calculated only from patients admitted to dedicated orthopedic units though some patients were housed in several other units of the hospital. Additionally, surgeons were uniquely responsible for determining patient recruitment and selection; the surgeons informed us approximately a year ago that their selection criteria for these procedures were evolving and they were no longer performing hip and knee surgeries on patients with a BMI greater than 40. Still, the distribution of types of procedures and ASA classification were consistent throughout the year. We are aware, in fact, of orthopedic surgeons having additional criteria for patients who received care under the PSH model, including cardiac clearance from the patient's primary care physician or cardiologist. This required clearance often demanded testing to be performed by the patient's primary care physician/cardiologist which was transmitted to our electronic medical record.

Table 1. Patient distribution for PSH patients in 2015 (Number of patient, types of surgery, percentage of ASA III and IV patients did not change significantly over the course of the 4 quarters)

\begin{tabular}{lllll}
\hline & Quarter 1 & Quarter 2 & Quarter 3 & Quarter 4 \\
\hline Number of patients & 379 & 321 & 332 & 324 \\
Hip & $38 \%$ & $33 \%$ & $43 \%$ & $41 \%$ \\
Knee & $42 \%$ & $51 \%$ & $44 \%$ & $43 \%$ \\
Shoulder & $20 \%$ & $16 \%$ & $13 \%$ & $16 \%$ \\
ASA III & $59 \%$ & $51 \%$ & $56 \%$ & $54 \%$ \\
ASA IV & $3 \%$ & $3 \%$ & $3 \%$ & $3 \%$ \\
\hline
\end{tabular}

Table 2. Changes in number of images, tests, blood units transfused and LOS over four quarters of 2015

\begin{tabular}{llllll}
\hline & Quarter 1 & Quarter 2 & Quarter 3 & Quarter 4 & $\Delta$ From Q1 to Q4 \\
\hline Imaging Per Case & 6.80 & 6.00 & 6.70 & 6.10 & $9 \%$ decrease \\
Tests Per Case & 6.1 & 6.0 & 5.8 & 4.7 & $22 \%$ decrease \\
Total Units of Blood Cells Transfused & 39 & 21 & 34 & 5 & $87 \%$ decrease \\
Length of Stay (LOS) & 3.35 & 2.97 & 3.04 & 2.99 & 0.4 day reduction in LOS \\
\hline
\end{tabular}



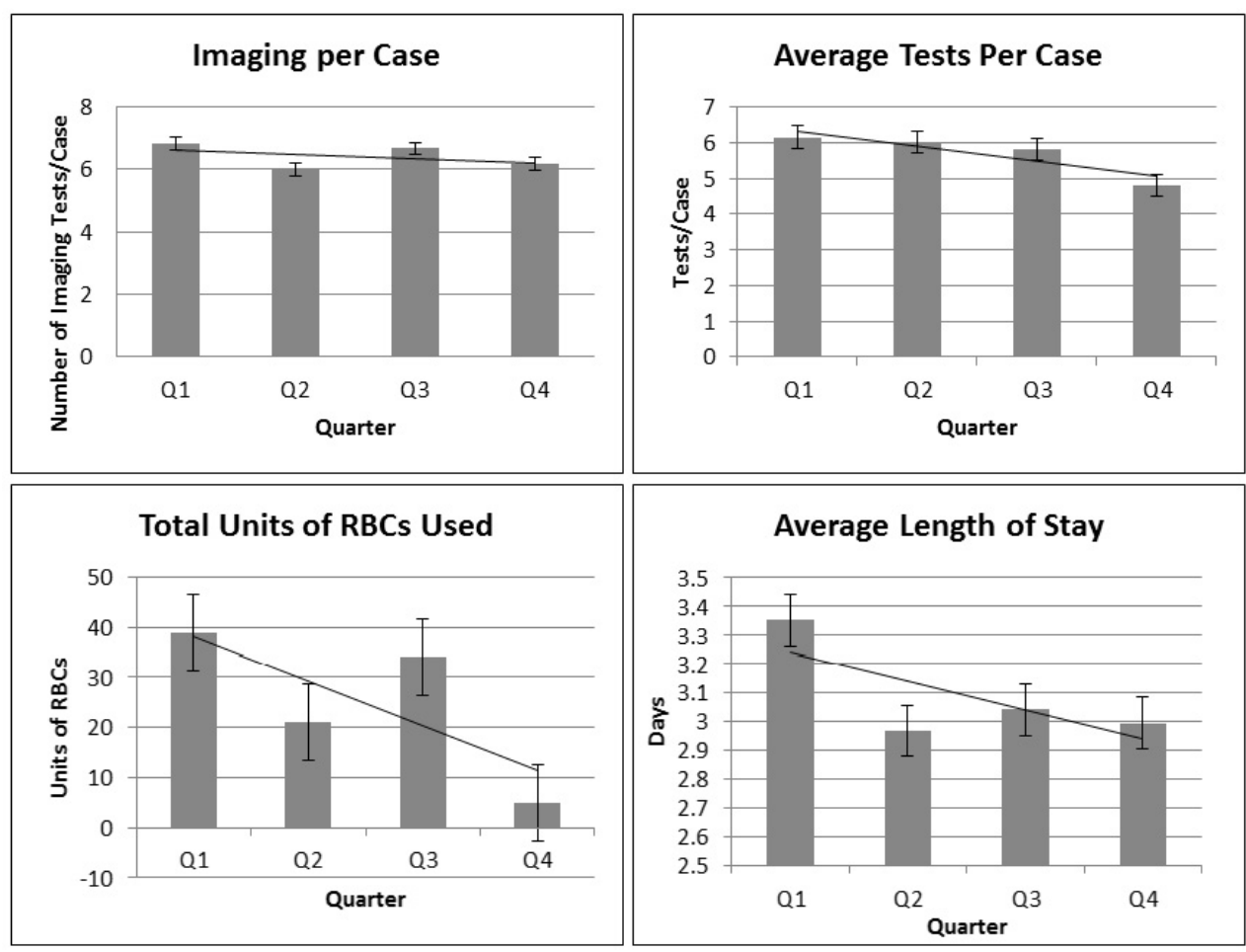

Figure 1. Reduction of resource utilization for major joint replacement at TGH in 2015 per quarter (A) $9 \%$ reduction in imaging per case; (B) $22 \%$ reduction in average tests per case; (C) $87 \%$ reduction in total units of red blood cells $(R B C s)$ used; (D) Decrease in length of stay for patients admitted to orthopedic ward (Error bars note standard error).
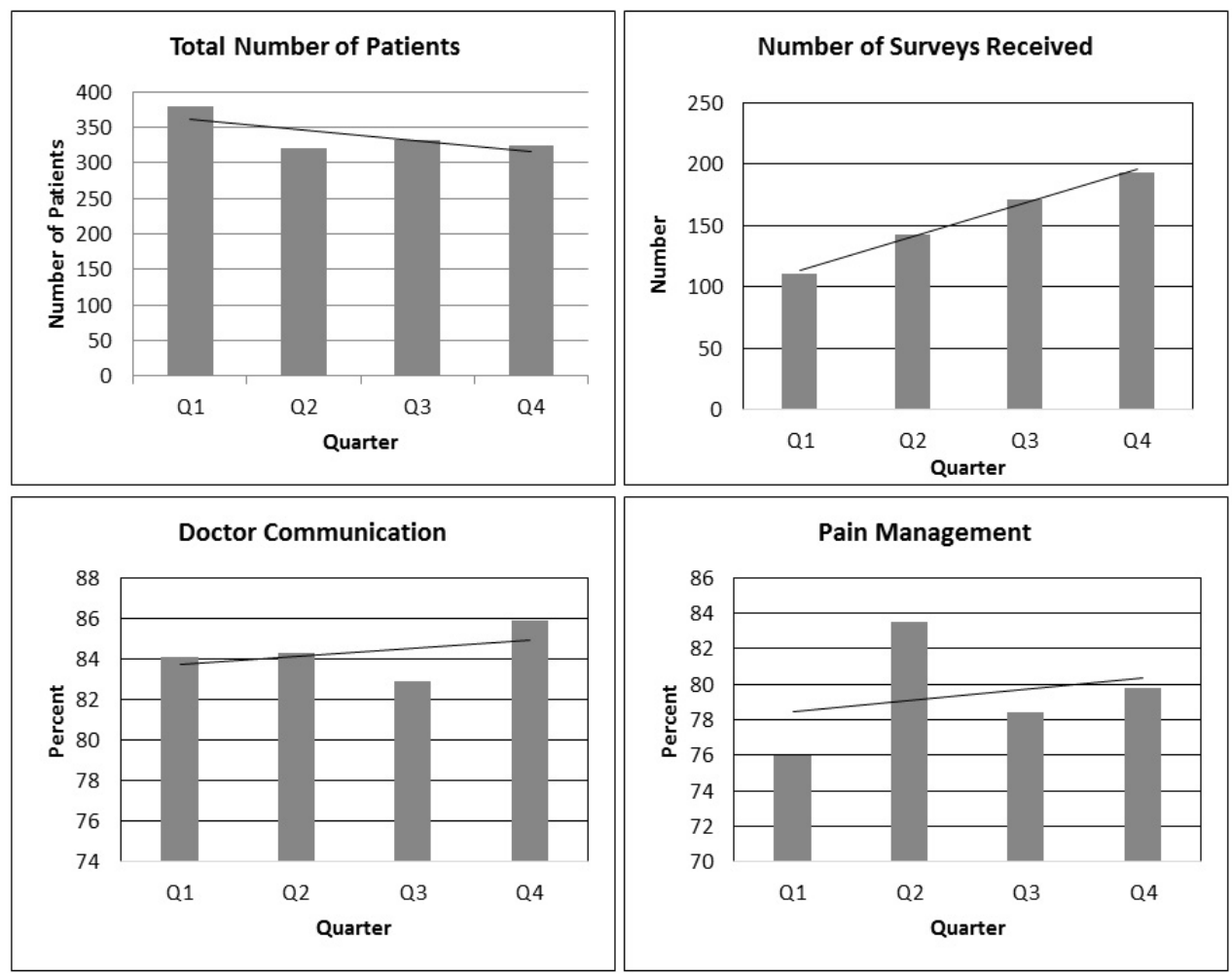

Figure 2. Patient satisfaction indices per quarter

Significant improvement in paint management scores and doctor communication parameters were noted at TGH in 2015 from quarter to quarter (Error bars note standard error). 
Anesthesiologists, in their traditional role, have a limited impact on a hospital's performance; but, in their role as perioperativists, anesthesiologists can play a pivotal role in promoting compliance for practices that require coordination across departments and throughout the preoperative, intraoperative, and postoperative periods. ${ }^{\left[{ }^{8-10]}\right.}$ The changes observed during the 12-month period were slow and required coordination across departments: rapid changes were only observed in the area of blood transfusion after a policy decision was implemented.

\section{Conclusions}

The PSH model is comprised of numerous small modifications to patient care which collectively contribute to the observed changes in patient outcomes; consequently, we cannot pinpoint the observed improvements to a single alteration within this multifactorial approach. As evidenced by our data, the transition was gradual since practices in the PSH model affect a large cohort of healthcare providers: from nursing staff and physicians to administrators and financial personnel. Still, the direction is such that we are able to concurrently utilize fewer resources and improve patient satisfaction.

\section{CONFlicts OF INTEREST Disclosure}

The authors declare they have no conflict of interest.

\section{REFERENCES}

[1] Schweitzer M, Fahy B, Leib M, et al. The Perioperative Surgical Home Model. ASA Newsletter. 2013; 77(6): 58-9.

[2] Holt NF. Trends in healthcare and the role of the anesthesiologist in the perioperative surgical home - the US perspective. Current Opinion in Anesthesiology. 2014; 27: 371-376. PMid: 24780942. https://doi.org/10.1097/ACD.0000000000000064

[3] Berwick DM, Nolan TW, Whittington J. The triple aim: care, health, and cost. Health Aff (Millwood). 2008; 27(3): 759-769. PMid: 18474969. https://doi.org/10.1377/hlthaff.27.3.759

[4] Stiefel M, Nolan K. A Guide to Measuring the Triple Aim: Population Health, Experience of Care, and Per Capita Cost Cambridge, Massachusetts: Institute for Healthcare Improvement; 2012. IHI Innovation Series white paper.

[5] Kain ZN, Vakharia S, Garson L, et al. The perioperative surgical home as a future perioperative practice model. Anesth Analg. 2014; 118(5): 1126-1130. PMid: 24781578. https://doi.org/10.121 3/ANE. 0000000000000190
[6] Vetter TR, Goeddel LA, Boudreaux AM, et al. The Perioperative Surgical Home: how can it make the case so everyone wins? Anesthesiology. 2013; 119(6): 1261-1274. PMid: 24005578. https: //doi.org/10.1097/ALN.0b013e3182a8e9e6

[7] Mangar D, Schweitzer MP, Ching YH, et al. The Impact of An Anesthesiology-Centered Preoperative Testing Protocol on Hospital Expenses and Adverse Events: A Before and After Retrospective Cohort Study. International Journal of Medical Science and Clinical Inventions. 2016; 3(3): 1646-1653.

[8] Huang J, Schweitzer M. The perioperative surgical home: what anesthesiologists need to do. J Med Pract Manage. 2014; 29(4): 235-237. PMid: 24696962.

[9] Garson L, Schwarzkopf R, Vakharia S, et al. Implementation of a Total Joint Replacement-Focused Perioperative Surgical Home: A Management Case Report. Anesthesia \& Analgesia May 2014. 2014; 118(5): 1081-1089. https://doi.org/10.1213/ane.00000000 00000191

[10] Warner M. Who better than anesthesiologists? The $44^{\text {th }}$ Rovenstine Lecture. Anesthesiology. 2006; 104: 1094-101. PMid: 16645463. https://doi.org/10.1097/00000542-200605000-00027 